\section{Limitantes didácticas y tecnológicas en la enseñanza universitaria en época pandémica}

Didactic and technological limitations in university educativon in pandemic times

\section{Pablo Alberto Parra Silva* Daniel Alberto Parra Gavilanes*}

\section{RESUMEN}

El propósito del estudio busca evidenciar la caracterización de la experiencia pedagógica virtual universitaria, en una circunstancia apresurada de aplicación de las clases virtuales. El objeto de estudio es la experiencia pedagógica virtual. El problema de investigación surge de la manera en que se ha abordado el desafío de la enseñanza virtual, desde la perspectiva pedagógica y didáctica en la Universidad Técnica Estatal de Quevedo, a partir del año 2020. El objetivo es considerar el desafío de la aplicación pedagógica virtual, la utilización didáctica docente y el acceso tecnológico, para una enseñanza de calidad, en la universidad, durante este período de pandemia. Se toma como muestra a doscientos treinta y cuatro estudiantes de los primeros semestres de distintas carreras universitarias, aplicando una encuesta cerrada, acorde al proceso de encuadre de las asignaturas a cargo de los investigadores. Se trata de una investigación argumentativa documental, cuyos resultados servirán como aporte significativo a un proyecto de investigación relacionado con los desafíos y limitaciones en la enseñanza virtual. Los resultados que se aspiran buscan proponer una nueva perspectiva pedagógica y metodológica institucional; estructurar mecanismos tutoriales de sustentación pedagógica para docentes de distintas profesiones; actualización de conocimientos pedagógicos, con aporte a la didáctica y a la utilización tecnológica virtual; la estructuración de

\footnotetext{
*Master, Universidad Técnica Estatal de Quevedo, pparra@uteq.edu.ec https://orcid.org/0000-0002-784I-758I

*Master, Universidad Técnica Estatal de Quevedo, dparra@uteq.edu.ec https://orcid.org/0000-0002-0645-2767
}

\section{REVISTA TECNOLÓGICA ciencia y educación Edwards Deming}

ISSN: 2600-5867

Atribución/Reconocimiento-NoCo mercial- Compartirlgual 4.0 Licencia Pública Internacional — CC

\section{BY-NC-SA 4.0}

https://creativecommons.org/licenses /by-nc- sa/4.0/legalcode.es

Editado por: Tecnológico Superior Corporativo Edwards Deming Enero - Julio Vol. 5 - I - 202 I https://revista-edwardsdeming.com/index.php/es e-ISSN: 2576-097I

Recibido: 02 Marzo 2020

Aprobado: 4 Diciembre, 2020

Pag 13-28 
manual instructivo psicopedagógico institucional; $y$, la socialización de los resultados del proyecto.

Palabras clave: Didáctica, Enseñanza, Metodología, Pedagogía virtual.

\section{ABSTRACT}

The purpose of the study is related to the expectation of showing the characterization of the virtual university pedagogical experience, in a hurried circumstance of application of virtual classes. The object of study is the virtual pedagogical experience. The research problema arises from the way in which the challenge of virtual teaching has been approached, from the pedagogical an didactic perpective in the State Technical University of Quevedo,from the year 2020. The objective is to consider the challenge of the pedagogical application virtual teaching, teaching didactic use and technological Access, for quality teaching, at the university, during this period of pandemic. Two hundred students from the first semesters of different university careers are taken as a sample, applying a closed survey, according to the process of framing the subjects in charge of the researchers. It is an argumentative documentary investigation, the results of which will serve as a significant contribution to a research project related to the challenges and limitations in virtual teaching. The aspired results seek to propose a new institutional pedagogical and methodological perspective; structuring tutorial mechanisms of pedagogical support for teachers of diferente professions; updating pedagogical knowledge, with a contribution to teaching and the use of virtual technology; the structuring of a institutional psychopedagogical instruction manual; and, the socialization of the projects results.

Keywords: Didactics, Methodology, Teaching, Virtual Pedagogy

\section{INTRODUCCIÓN}

Las distintas instituciones de educación superior, tienen sus propias narrativas del proceso de educación virtual, que debió asumir la universidad en la época de pandemia, comprendida entre los años 2020 y 2021 . El problema de investigación responde a la pregunta: ¿Dónde radica el desafío de la aplicación pedagógica virtual, la utilización didáctica docente y el acceso tecnológico, para una enseñanza de calidad, en la Universidad Técnica Estatal de Quevedo-Ecuador, durante el período 2020-202I?

El estudio plantea tres objetivos específicos, que son: Señalar la manera en que se ha asumido la enseñanza virtual, desde la perspectiva pedagógica y didáctica en la Universidad Técnica Estatal de Quevedo, a partir del año 2020. Porque la imprevista presencia de la educación virtual al inicio del año 2020, dio lugar a que la universidad asumiera el proceso de enseñanza-aprendizaje, de una manera improvisada, pero al mismo tiempo decidida a enfrentar ese reto. Para lo cual, la docencia asumió el cumplimiento de su tarea académica, con los conocimientos pedagógicos y didácticos de que disponía, apoyados por los recursos y herramientas ofrecidos por la institución de educación superior, que ya venía dando sus resultados desde la esfera de la presencialidad. 
Un segundo objetivo específico busca: Describir las características pedagógicas, didácticas y tecnológicas implementadas por los docentes en la Universidad Técnica Estatal de Quevedo, a partir de mayo 2020, hasta el presente. Porque se trata de describir realmente la manera como asumieron los docentes sus funciones pedagógicas, didácticas y tecnológicas, implementadas en la mayoría de los casos por primera ocasión para la educación virtual. Para que se determine con precisión si realmente los estudiantes recibían eficientes y adecuados recursos pedagógicos, didácticos y tecnológicos, por parte de los docentes.

Un tercer objetivo específico se centra en: Analizar las limitaciones pedagógicas, didácticas y tecnológicas, que se han asumido para enfrentar el reto de enseñanzaaprendizaje en la Universidad Técnica Estatal de Quevedo, con relación al año 202I. Porque en la enseñanza-aprendizaje se debe hacer un estudio minucioso y detallado del exitoso cumplimiento de las acciones pedagógicas, didácticas y tecnológicas, que se ha asumido para enfrentar el reto de la enseñanza-aprendizaje en línea o virtual. Para evidenciar que se ha asumido el reto del proceso educativo universitario con un buen nivel, evidenciando las limitaciones que han acontecido, a partir de lo cual se busca proponer un plan de acción y de actualización de conocimientos, a partir del año 2021 . No podemos soslayar el hecho de que la docencia universitaria, carece de una formación previa en cuanto a fundamentos de pedagogía, didáctica y tecnología. Indudablemente, hay segmentos del claustro universitario que tienen notables conocimientos de estos campos; sin embargo, la gran mayoría de docentes ingresan a las universidades aplicando un concurso de merecimientos o como contratados, pero en la mayoría de los casos como expertos profesionistas, sin haber confirmado su idoneidad como expertos pedagogos. Una gran mayoría de profesores, simplemente se limita a replicar lo que a ellos también les enseñaron otros profesores en las aulas de clases, mantienen incluso su estilo, sus estrategias y su empírica actitud docente.

Esta apreciación encaja con el planteamiento problemático de Carlos Álvarez de Zayas, en su "Escuela en la vida", donde sostiene que algunas deficiencias son: que la actividad laboral no siempre se organiza como parte del proceso docente-educativo, no tiene objetivos y contenidos precisos, no hay una integración en la formación académica, los profesores no siempre se sienten responsabilizados con el desarrollo de la actividad laboral de quien instruyen, entre otros aspectos (Álvarez de Zayas, 1999) .

En tal sentido la Pedagogía "es la ciencia que tiene como objeto de estudio el proceso formativo", con direccionamiento científico, buscando alcanzar niveles de calidad y excelencia. Mientras por otra parte la didáctica, que estudia el proceso educativodocente, se dirige a la preparación del sujeto para la vida, de un modo sistémico y eficiente.

El actual contexto exige que el proceso de enseñanza-aprendizaje universitario propicie el protagonismo de los estudiantes. Prestando mayor atención a la preparación de la clase, asumiendo estrecha relación con el proceso, potenciando la participación activa. El docente debe emplear medios de enseñanza, organizar y evaluar el proceso, en correspondencia con el método de enseñanza-aprendizaje (Hernández \& Infante, 2016). 
Pero en la práctica se observan insuficiencias en la utilización adecuada del método, donde la gran mayoría de profesores da disposiciones, utiliza vídeos ilustrativos, envía trabajos a los estudiantes, éstos exponen la clase, hay una ambigua explicación y se limitan a incorporar evaluaciones automatizadas, a través de plataformas tecnológicas que se encargan automáticamente de calificar las respuestas, que se limitan a un par de estilos de "pruebas objetivas" y se resolvió el semestre.

La adquisición de los conocimientos, el desarrollo de habilidades y valores, se asocia al método que selecciona y emplea el docente, en dependencia del propósito trazado. Evidenciando la necesidad de prestar mayor atención a la concepción didáctica de la clase.

Los métodos, expositivo, investigativo y de trabajo independiente, son esenciales en la transmisión de conocimientos. Pero no implica que el estudiante adopte una actitud meramente receptora o repetitiva de lo que otros autores "dicen". Es necesario que el cúmulo de saberes sea comunicado claramente a los educandos, planificadamente y garantizando la participación activa de los estudiantes en la clase.

Los métodos, investigativo y de trabajo independiente, implican el desarrollo de la actividad cognitiva, a través de la ejecución de tareas orientadas, buscando fuentes determinadas, con trabajo independiente, honesto, comprometido y dentro de las acciones planificadas a través del sílabo de cada asignatura.

Los estudiantes en su proceso de trabajo independiente, también requieren de planificación, orientación, control y evaluación por parte del docente. Se trata de favorecer la autonomía, pero el docente debe verificar con certeza que sus resultados converjan en alcanzar el desempeño esperado, bajo su conducción y guía.

El método de trabajo independiente debe estar perfectamente comprendido, para que pueda contribuir al desarrollo de la personalidad del estudiante, aportando a su independencia cognoscitiva. Sin embargo, el método de enseñanza-aprendizaje, como camino para desarrollar los contenidos que se imparten, requieren de la utilización de medios de enseñanza idóneos, que evidencien la relación profesor-estudiante, en un trabajo planificado, orientado, controlado y evaluado por el docente.

La clase encuentro más adecuada, será entonces aquella en la que el profesor muestra todas sus estrategias psicopedagógicas, andragógicas, didácticas y en el caso de la educación virtual, tecnológicas. Solo la participación consciente de los estudiantes en su aprendizaje, contribuirá al desarrollo integral de sus conocimientos y de su estructura como individuo.

La didáctica centrada en el estudiante implica deliberadamente la utilización de concepciones, modelos, alternativas, estrategias y métodos aplicados idóneamente en el proceso de enseñanza-aprendizaje, que van mucho más allá de la utilización mecánica de plataformas y herramientas tecnológicas, que han distanciado mucho más el papel del docente-tutor. La pedagogía enfrenta el reto de que el estudiante produzca el conocimiento, desarrollando un pensamiento reflexivo y crítico desde el punto de vista cognoscitivo. 
Ahora más que nunca, en ese período de educación virtual, debería estar en el repertorio de los docentes elementos didácticos basados en proyectos, teorizados a partir de adecuadas problematizaciones, con casuísticas acordes a la carrera profesional, con discusiones, dinámicas grupales y aprendizaje colaborativo, donde se combinen técnicas participativas, analogías, demostraciones, mapas conceptuales, junto a todas las herramientas que la tecnología pudiera facilitar.

La improvisada gestión educativa a distancia, ha tomado el pulso a los docentes universitarios en cuanto a sus verdaderos conocimientos pedagógicos y aportaciones didácticas. Las autoridades académicas se han limitado a supervisar este proceso, con un alejado mecanismo de evidenciar las clases sincrónicas y asincrónicas, de una manera muy superficial y sin sujeción a un modelo educativo específico.

Se está eludiendo el hecho de que quienes reciben realmente la ilustración de los profesores, son una cantidad significativa de estudiantes, quienes a su modo, tampoco han sido plenamente capacitados en desarrollar aspectos específicos como la metodología de la investigación científica, la adecuada estructuración gramatical y sintáctica en ensayos y trabajos, la responsabilidad de la utilización de referencias bibliográficas precisas, el uso adecuado de gráficos y láminas para divulgación académica, el responsable trabajo en equipo, la dedicación del tiempo como parte de la iniciativa autónoma individual.

Con el advenimiento del aula virtual, de la tecnología y de otros nuevos dialectos elearning, plataforma tecnológica, APP, aprendizaje invertido, competencias digitales, espacios virtuales, sitios web, software educativo, TIC, criptoMobileApp, alfabetización tecnológica, brechas digitales, herramientas digitales, aplicación framework, ciberadicción, ciberbullying, entre otras decenas de palabrerías tecnologizantes, nos encontramos de un momento a otro volcados en un nuevo proceso de enseñanzaaprendizaje, en todos los niveles educativos.

Lo que más preocupa, es que se está procurando una notable capacitación en el uso de la tecnología, especialmente a docentes que nunca antes habían utilizado estas herramientas para un trabajo virtual; o bien, su infrecuente uso no cubría las expectativas y exigencias que ahora se requiere; así como también, durante muchos años, esos profesores "dictaban" sus clases a puerta cerrada en un aula de cuatro paredes. La ausencia de un verdadero proceso de capacitación psicopedagógica, es lo que realmente preocupa.

Muchos creen que es suficiente con haber aprendido dos 0 tres mecanismos procedimentales, "para dar las clases"; pero no se han nutrido de un conjunto de elementos conceptuales, epistemológicos, metodológicos, estratégicos, didácticos, pedagógicos, psicológicos, que además comprometan al mejoramiento de la estructura axiológica y humana, que debería caracterizar al profesor de la nueva era.

Esto va a conducir a cuatro opciones: la primera, a que todo el mundo apruebe, porque la flexibilidad que exige el momento actual, podría conducir a un facilismo extremo, que inutilice cualquier plan de estudios o de crecimiento académico de los estudiantes. En este mismo contexto, estará ubicado el profesor que no se hace problema y que 
simplemente "copia y pega", sin ningún tipo de preocupación por la ética académica, pues el asunto es cumplir con todas estas nuevas exigencias institucionales.

La segunda opción, que parece va a desarrollarse, es la del profesor que traduzca exactamente como era durante todos sus años de docencia, convirtiendo la clase virtual en una repetición del pasado, con los mismos elementos de juicios, tonalidades, muletillas, modismos, discursos, griteríos, que hacía cuando era profesor en el aula presencial. Ahí se percibe la presencia de aquel profesor de otras épocas, omnipotente, frívolo, insípido, de escaso hablar, de pronunciación incomprensible, de clases improvisadas y de retórica fuera de contexto. Ese profesor de difícil acceso, con el cual es casi imposible lograr una conversación interactuante, sino simplemente acostumbrarse a ser receptores pasivos de su sabiduría. Y, por cierto, temerle sobre todo en las calificaciones.

La tercera opción que se podría producir, es la del profesor tecnócrata, experto, erudito y autodidacta de TIC, que es muy intelectual, conocedor de los últimos avances de la tecnología, "sorprendedor" con nuevos enlaces y aplicaciones informáticas, que dejan en ascuas a los estudiantes, a quienes no les va a quedar otra alternativa que pagar a expertos para que les ayuden a resolver todas las tareas y demandas del docente. Si se puede decir algo más, será el profesor dominante, a quien nadie termina de comprender sus explicaciones, pero que deja sin aliento y sin horas de sueño a cualquiera.

Una cuarta opción, es la de los docentes que van a entender que el proceso de enseñanza-aprendizaje es mutuo. Es colaborativo y compartido. Implica una actitud flexibilizada, humana, personalizada, comunicacional, interactiva, afectiva y emocional. Donde utilizando estas condiciones, se traduzca positivamente la información, que de otra manera se podría presentar demasiado fría o indiferente, al estar simplemente frente a un ordenador y una pantalla que visualice un rostro con el nombre de una persona. Dar valor al individuo que está interactuando, transmitiéndole a su vez un conjunto de conocimientos y valores, que fortalezcan, modifiquen y optimicen sus propios elementos conceptuales, comportamentales, intelectuales y de desarrollo humano.

Estudios anteriores a la pandemia, visualizaron los entornos educativos universitarios, con una buena práctica educativa, la mediación de las tecnologías digitales y las redes sociales, además de dictaminar el rigor del trabajo, su sustento teórico y la innovación de las experiencias y modelos educativos. Uno de los principales aspectos considerados fue el de la equidad: para el mejoramiento de la distribución social de los resultados educativos, la cobertura de la enseñanza y la adquisición de competencias con miras al siglo XXI.

Pero significativamente se pensó en la eficiencia; esto es, en el mejoramiento del sistema educativo o del aula, procurando la disminución de la repetición del curso, evitar el rezago o el abandono estudiantil. Se escribió mucho sobre la alfabetización digital en el proceso educativo (Flores, Díaz B., \& Rigo, 2016).

La Universidad Técnica Estatal de Quevedo (UTEQ)-Ecuador, asumió a partir de abril de 2020, la incorporación al proceso de educación virtual, ante la inesperada condición 
pandémica que amenazaba la salud humana y exigía el distanciamiento. Ninguna institución educativa superior esperaba que la educación virtual se acelerara con tal brevedad. Sin embargo, uno de los beneficios de la gestión administrativa en la UTEQ, fue la disposición de una adecuada plataforma de gestión académica (SGA), que junto a otras aplicaciones tecnológicas (Classroom, Meet, Zoom), permitieron que el improvisado proceso educativo quede en manos de los docentes, así como de los estudiantes y sus respectivos apoyos familiares.

Siendo que la educación superior es gratuita, nadie incurrió en la sugestiva obligatoriedad de conseguir las herramientas tecnológicas para resolver el problema educativo del mundo en el 2020. El estudiante y el profesor se vieron en el apremio de resolver conexiones de internet, obtener equipos de trabajo de buen nivel para acceder a la información que se aspiraba transmitir. En definitiva, asumir el costo de ser educador y de ser educando universitario.

A la condición de limitación y de notable carencia o dificultad, se le dio un remedio infalible llamado "flexibilidad académica". Las autoridades académicas y los docentes deberán ser flexibles y creativos para asegurar la consecución de los objetivos de aprendizaje de los estudiantes en estas difíciles circunstancias, especialmente en todo lo que concierna a la evaluación (PUCE. Pontificia Universidad Católica del Ecuador., 2020). Con ello quedaba remediado que el estudiante no tuviera adecuadas herramientas de trabajo, puesto que su condición socioeconómica le impedía su obtención. Se superaba en nombre de dicha elasticidad, el desconocimiento que el profesor y el estudiante tengan respecto al uso de instrumentos tecnológicos. A lo blando del panorama se incorporaba que ahora no era necesaria ni obligatoria la asistencia virtual a clases; puesto que el profesor tenía la obligación de subir de manera asincrónica las clases. Cuestiones que incluso eran disposiciones y regulaciones nacionales, que evitaba la presión social ante el Estado, de proveer por ejemplo de gratuidad en la conexión de internet o fácil acceso a la adquisición de equipos de trabajo (laptops, tablets, computadoras, teléfonos celulares). La ley exigía flexibilidad, pero a la par la educación universitaria asumía su mediocridad.

"Una concepción general sobre el aprendizaje representa una herramienta heurística indispensable para el trabajo diario de los docentes; les brinda una comprensión de los complejos y diversos fenómenos que tienen lugar en el aula $y$, por lo tanto, un fundamento teórico, metodológico y práctico para planificar, organizar, dirigir, desarrollar y evaluar su práctica profesional, perfeccionándola continuamente. Todo esto constituye un requisito básico para que el educador pueda potenciar, de manera científica e intencional - y no empírica o intuitivamente - los tipos de aprendizaje necesarios, es decir, aquellos que propician en sus estudiantes el crecimiento y enriquecimiento integral de sus recursos como seres humanos, en otras palabras, los aprendizajes desarrolladores" (López, Chou, \& Granado, 2017).

La figura del profesor-tutor vuelve a redimensionarse en la educación virtual, pues le corresponde conducir a la educación universitaria a un más alto nivel, como un agente de cambio, que acompañe al proceso de desarrollo integral de la formación profesional 
y personal del estudiante (Herrera R., 2008). Pero, sobre todo, despertando empoderamiento en el proceso de enseñanza-aprendizaje, apoyando y coordinando la prevención de dificultades de aprendizaje o de conductas que obstaculicen el buen desempeño del aula.

La súbita transformación del hogar del estudiante en aula de clase, así como el encuentro de un espacio en la casa del profesor, para convertirla en aula virtual, generó distintas disposiciones en el entramado laboral y en la vida cotidiana de todos. Las disposiciones personales y el uso de Tecnologías de la Información y Comunicación (TIC), junto a la obtención de dispositivos digitales, conectividad a internet, utilización de herramientas tecnológicas, se entrelazaron con el desarrollo de saberes disciplinares, de actitudes pedagógicas, consideraciones a la dimensión didáctica, transición urgente a superar la brecha digital.

Para abril/2020 se inició en la UTEQ un curso de capacitación para el uso de herramientas tecnológicas. Se partió de la información del Reglamento de Régimen Académico (2019), especialmente de los artículos 26, 28, 68, 70; donde se refería a la intervención directa del docente en forma presencial o virtual, sincrónica o asincrónica, a través de conferencias, clases, seminarios, talleres y proyectos de aula.

También se inició enfocando la importancia de la tutoría personalizada, el acompañamiento para superar las principales dificultades. Pero también se refirió al aprendizaje autónomo, el uso de plataformas digitales, los ambientes en línea y a distancia. Se hizo énfasis en sensibilizar la forma de ser del docente, utilizando las ayudas tecnológicas, sin dejar de "ser humanos". Procurando desarrollar conocimientos, experiencias, estrategias metodológicas, que permitan que el estudiante se desarrolle de manera significativa, sin perder la rigurosidad académica y siendo el propio gestor del aprendizaje.

El I5 de mayo/2020, teniendo como conferencista invitado al Dr. Manuel Herrera, de la Universidad Internacional de La Rioja, se expuso que la UTEQ había despuntado el número de matriculados de 8836 a 9030 , superando la base de matrícula prevista. Se habló de una mutación, de un cambio radical en nuestras costumbres, especialmente en la forma de relacionarnos con nuestros hábitos de trabajo, con nuestras formas de usar el tiempo libre y con nuestra forma de educarnos.

Se planteó que las nuevas tecnologías llegaron a quedarse; pero también se sustentó, que la educación on line no es la educación presencial hecha a través de internet. Que implicaba una metodología y estrategias, que permitan que el estudiante adquiera competencias necesarias para su carrera universitaria. Pero igualmente, se anticipó la incorporación "de píldoras informativas de diez o quince minutos", que no serían otra cosa que "clases enlatadas" con el objeto de completar lo que no se había podido lograr en la clase virtual sincrónica.

Se planteó la importancia del acompañamiento, puesto que el estudiante está solo en su casa, su campus virtual es su computador o su teléfono. Mediante el acompañamiento, cada estudiante debería sentir a su profesor como un tutor, que es un coaching, "un ángel de la guarda", que se conecta con el estudiante de manera continua y consistente. 
Para lo cual el profesor tiene que estar capacitado para el uso de las herramientas digitales.

De esta manera el profesor no se podría limitar "a dictar una clase", sino a dinamizar los conocimientos, generar debates, impartir generalidades, mientras el estudiante profundizaba en los temas, con otras herramientas y recursos. Propiciar la autonomía del estudiante, no implicaba que el profesor desapareciera del escenario contextual de la clase, sino convertirse en un instrumentalizador de conocimientos.

Urgentemente se ejecutaron exposiciones magistrales a través de los propios docentes expertos en informática, donde se provocó información en torno a charlas Meet o Zoom, instalación de programas, enlaces tipo Facebook o whatssapp, OBS, Google y otros navegadores. Provocándose irremediablemente una carga informática, que dejó a muchos docentes en ascuas y con una necesidad de autocapacitarse inmediatamente.

Para octubre/2020 se capacitó a los docentes de la UTEQ, en relación con procesos de generación de recursos académicos. Se dispuso una exigua información sobre software para grabación y transmisión de vídeos por internet, creación y edición de vídeos, programas para comprimir y preparar vídeos MP4 (Open Broadcaster Software, OpenShot video editor, HandBrake), como aplicaciones libres de código abierto. Con estos recursos se esperaba que los docentes pudieran desarrollar actividades asincrónicas, para ser subidas a través del Sistema General Académico (SGA), cuestión que a la larga resultó un tanto complejo, porque el peso de los archivos obligaba a reducir a un mínimo en tiempo y en espacio, la información emitida por el profesor.

De igual manera, para octubre 2020, la Asamblea del Sistema de Educación Superior Ecuatoriana (ASESEC), condujo una perspectiva de aprendizaje dirigida a transformar la educación, buscando tomar decisiones y hacer operativo el aprendizaje, proporcionando criterios para la formación en línea. Pero se señaló que las pautas básicas empiezan con la formación del docente, quien se dijo, ha de permanecer en constante formación y descubrimiento de metodologías y modelos pedagógicos, que le permitan trasladar información adecuada a los estudiantes (UNIR). Se trataba pues, de lograr que el profesor maneje las herramientas tecnológicas que manejan los estudiantes, moviéndonos por igual en la era digital.

Pero algo significativo, fue que se hizo énfasis en el acompañamiento al estudiante. La confianza en la institución y la sensación de apoyo, para motivarlos al trabajo y al seguimiento continuo de la programación académica. Sin embargo, fue muy poco fortalecido este aspecto en el resto de la capacitación. Se privilegió la evaluación continua y el uso de rúbricas, alineadas a las conexiones on line y los soportes tecnológicos.

Para noviembre/2020, se proporciona un taller de CEDIA, sobre "Calidad y excelencia educativas", dirigido por la Dra. Adriana Arnao, que se fundamenta en la calidad, la excelencia, la certificación y la acreditación-habilitación. Todo aquello, con miras a lograr la calidad educativa, pero con una retórica ecuménica, que se dirigía en su mayor parte a medir resultados a través de la evaluación del aprendizaje. Entonces, el fondo de la ilustración era la capacitación a realizar reactivos, con pautas de selección múltiple, de 
jerarquización y de correlación. Lo que pareciera se convirtió en un arma tecnológica, para actualizar el antiguo terror de los exámenes presenciales.

En este punto era de vital importancia el fortalecimiento de la pedagogía y la ilustración didáctica en los docentes universitarios de la UTEQ. De lo contrario, el seminario solo serviría de adoctrinamiento y refinamiento de viejas tendencias de dominio absolutista del profesor sobre los resultados de aprendizaje del estudiante.

Se confirmó este aprendizaje con el curso de elaboración de reactivos, a través del SGA, que se realizó el 4 de noviembre/2020. El SGA constituye en una plataforma que recoge las evidencias del trabajo académico de los profesores de la UTEQ. Ahí se estructura la información que debe recibir el estudiante, pero sobre todo los procesos de enseñanzaaprendizaje evidenciados por sendos archivos en pdf, que deben ser registrados en cada clase; precisamente, controlando la asistencia puntual del profesor, a manera de un marcador digital como forma mecánica de evaluar el accionar del docente. Su información sirve para el control directamente de parte de coordinadores y autoridades de la institución. El sílabo y el desarrollo de los contenidos académicos, deben quedar ilustrados como evidencia del proceso. Un enfoque del manejo instrumental de esta herramienta, pero sin ningún respaldo psicopedagógico como contraparte al proceso formativo del docente.

Resulta valedero e importante estudiar e interpretar las reacciones de la comunidad estudiantil y del profesorado universitario, en primer lugar, ante la transición a las clases virtuales; como también frente a los resultados de enseñanza-aprendizaje, que dimensionan el éxito o el fracaso de la educación on line, en relación al rol de los protagonistas (Carbajal L., 2020). Lo fundamental es que con presencia tecnológica o no, con presencialidad en el aula o con educación on line, lo importante es que se espera profesores capaces, con cierto ámbito de conocimientos en tecnología, pero sobre todo con elevada capacidad para la comunicación de saberes, a través de adecuadas estrategias pedagógica, didácticas, metodológicas, pero sobre todo con calidez humana.

La ampliación tecnológica en los espacios educativos, debe incorporar la inclusión educativa de grupos de población excluidos del estado de bienestar socioeconómico. No es suficiente pensar en las aulas virtuales como espacios de encuentro entre saberes disciplinares, didácticos y tecnológicos. Lo importante es una cultura institucional permeable que se transforma pedagógicamente, con la urgencia requerida por el sistema educativo, pero no depositando en las tecnologías y en el trabajo autónomo, la solución a las demás limitaciones ancestrales del proceso educativo, donde las principales carencias seguirán siendo aquellos aspectos ligados a la pedagogía y la didáctica del profesor universitario (De Luca, 2020).

\section{MATERIALES Y MÉTODOS}

Se ha planteado un tipo de investigación teórico-explicativa, cuyo propósito generar una interpretación teórica y práctica, relacionada con el proceso educativo de enseñanzaaprendizaje durante este periodo pandémico, acontecido entre el 2020 y el 2021 . Se 
pretende encontrar estrategias que puedan ser empleadas en el abordaje de la pedagogía, didáctica y tecnología, en el marco de la educación superior.

Para esto se utilizó una muestra de doscientas treinta y dos personas, estudiantes de distintos segmentos académicos de la UTEQ, que representan una muestra significativa, para objeto de esta investigación. Se desarrollo, con base a las hipótesis planteadas, siete ítems significativos que buscaban reflejar la apreciación del proceso, desde la perspectiva de los estudiantes beneficiarios de la educación virtual universitaria. Utilizando la plataforma virtual de Google institucional, se aplicó a los estudiantes vinculados a la tarea de los investigadores en su condición de docentes, lo que facultaba la inmediata y urgente respuesta a este trabajo, que viene a ser parte de una propuesta de investigación del equipo de trabajo.

La encuesta consta de seis preguntas, cuatro de las cuales son cerradas y dos abiertas; estas últimas con el propósito de establecer la existencia de nuevas variables, que puedan ser consideradas de utilidad en la interpretación y estudio de este tema.

\section{RESULTADOS}

Es significativo el resultado de la pregunta. Ante la hipótesis de saber si el abordaje pedagógico y didáctico de los docentes ha tenido un elevado nivel empírico y voluntarioso, que ha contrastado en los resultados académicos logrados, por la incoherencia y limitadas condiciones pedagógicas y didácticas de los docentes en general; es una magnífica aprobación de un $53,4 \%$ que admite que los docentes tienen una gran experiencia con la enseñanza en línea. Junto al $45,7 \%$ que consideran que los docentes han logrado cierto nivel de experiencia, en este mismo aspecto. Echa al traste la hipótesis en cuanto a estos resultados. Hecho que refleja probablemente, el enorme esfuerzo institucional y de cada docente, en lograr el cumplimiento del propósito innovador urgente de la educación virtual, sin haber tenido una experticia anterior, ni tampoco estar capacitados ampliamente los profesores para esta nueva forma de enseñanza.

Un reflejo altamente positivo se puede apreciar cuando los/las estudiantes señalan aspectos gratificantes en la enseñanza virtual. Uno de éstos, precisamente, una notable flexibilidad del proceso de enseñanza-aprendizaje, que vino a paliar las condiciones críticas con las que se iniciaron en este proceso los estudiantes. Seguida por la ejecución adecuada y precisa de un encuadre académico, que permite al estudiante empoderarse productivamente de la tarea. Se corrobora esto con un porcentaje significativo relacionado con la comunicación cordial y franca de los profesores; junto con un significativo uso de distintas herramientas tecnológicas para el trabajo on-line, proveniente de las condiciones previamente adquiridas por la gestión administrativa y académica de la universidad, que sirvieron de soporte para este tipo de resultado significativo y motivador para la enseñanza en línea. Debe destacarse el hecho, de que no existen evidencias de rechazo ni inconformidad en cuanto a la experiencia.

Se marca como un parámetro altamente significativo $(56,5 \%)$, de que uno de los retos más significativos logrados por los docentes universitarios, ha sido precisamente el 
cambio de la educación presencial a la enseñanza virtual. Frente a un $40,5 \%$, que señala como otra de las exigencias notables, la carencia de un servicio de internet estable y de calidad, circunstancia que no le corresponde asumirla a la universidad, sino que han tenido que sufragarla los estudiantes y los docentes; cuando en la realidad debiera ser una exigencia y obligación que debe asumirla el propio Estado ecuatoriano, en beneficio de la gratuidad del servicio de internet para la educación en el país.

Frente a la consideración de si era más conveniente y mejor la enseñanza presencial, un rotundo $75,3 \%$ señala que sí. Lo que advierte que tampoco significa un rechazo a la educación virtual, como lo confirma la siguiente pregunta de la encuesta, sino una necesidad gregaria que tiene el ser humano, de vincularse socialmente con el conglomerado, que como producto de esta etapa pandémica tuvo que alejarse y mantenerse desde sus propios hogares, sin un contacto presencial.

De ahí que la quinta pregunta que evalúa el sostenimiento de la educación virtual, como modalidad que podría coexistir con la presencialidad, le otorga un notable $88 \%$ de aceptación y aprobación. Es indudable, que la educación virtual vino a quedarse en la educación del 2021 .

Las otras dos preguntas, que son abiertas, permiten confirmar de manera significativa y evidente que los resultados de la educación virtual en la UTEQ, han salido airosas y con resultados altamente favorables, a pesar de que la argumentación teórica diga lo contrario. Es decir, los aspectos pedagógicos y didácticos, se han cumplido de manera altamente significativa, aunque que existen algunos eventos aislados, que debieran ser considerados como corregibles y mejorables.

La hipótesis de que los procedimientos de enseñanza-aprendizaje, han tenido limitadas condiciones didácticas y tecnológicas, que no han cubierto las expectativas académicas en la Universidad Técnica Estatal de Quevedo, no se cumple. Por el contrario, denota una franca y amplia aprobación de parte de la muestra estudiantil, objeto de la investigación.

Del mismo modo, la tecnología no tiene ninguna objeción, más allá que la repetida inconformidad con los servicios de internet, los cuales son ofertados por empresas particulares o públicas. Dejando como aprobada la hipótesis de que la construcción y diseño de recursos pedagógicos virtuales, vinculados a la didáctica y al adecuado entrenamiento de utilización tecnológica, incidirá en la optimización de la enseñanza en la Universidad Técnica Estatal de Quevedo.

\section{DISCUSIÓN}

Cuando estudios latinoamericanos refieren los impactos inmediatos de la pandemia en la educación superior universitaria, se dice que afloran a mediano y largo plazo, considerando además las acciones que toman los gobiernos para garantizar el derecho a la educación superior, frente a encarar la reapertura de las IES. Se predice los costes y las cargas financieras al estudiante, así como la continuidad del aprendizaje. Los efectos en el profesorado en cuanto a afectación laboral, especialmente del personal contratado (IESALC, 2020). La UTEQ, a través de sus directivos, logró superar este escollo gracias 
a una premeditada actitud organizacional, que evitó la disrupción del funcionamiento institucional; logrando, por el contrario, una estabilidad financiera y de gestión administrativa, que contrastó con los pronósticos del mundo. Igualmente, hubo una notable intervención de aporte significativo, gracias al empoderamiento docente, con relación a sus funciones sustantivas, con el cumplimiento cabal de sus roles, tal como lo describen los resultados evaluados en la investigación.

La reanudación de las actividades presenciales, será vista como una oportunidad de repensar el proceso educativo, confeccionar con más calidad el proceso de enseñanzaaprendizaje y generar nuevos mecanismos de concertación, para el avance institucional de manera óptima y sostenida por su acreditación nacional.

Elizabeth Rafael (20l3), planeaba que las nuevas tecnologías no tocan la muerte de los profesores, sino más bien el principio de su revaloración. A pesar de la resistencia al uso de la tecnología, el mundo entero se vio precisado a adoptar urgentes medidas de capacitación y de autoaprendizaje, logrando que cada docente se involucre de manera decidida y comprometida con su tarea; lo que también viene de la mano con la tarea de mayor responsabilidad y trabajo colaborativo y autónomo que debe desplegar el estudiante (Rafael S., 20l3). El cambio en el rol del docente, puede coincidir con cambios en la teoría del aprendizaje, donde la didáctica y la pedagogía, se vean fortalecidas grandemente.

El profesorado ha sido actor fundamental en la respuesta educativa ante la crisis sanitaria. No solo debiendo replanificar y adaptar los procesos educativos, ajustes metodológicos, reorganización curricular, diseño de nuevos recursos didácticos, diversificación de los medios, formatos y plataformas de trabajo; sino que también ha tenido que aprender a desarrollar actitudes de flexibilidad y apoyo socioemocional a los estudiantes, haciendo que la acción pedagógica deba enfrentar con éxito los retos de adecuar la oferta académica y los formatos pedagógicos, especialmente a estudiantes menos favorecidos socioeconómicamente.

Pero, además, la mayoría de países de América Latina han proporcionado a sus docentes la formación en el uso de herramientas de TIC, en niveles del 64\% en Brasil, $77 \%$ en Chile, 75\% en Colombia, 53\% en Argentina (CEPAL-UNESCO, 2020). Siendo tan breve e inconstante la capacitación al docente de la UTEQ, se vuelve magistral el éxito logrado en este primer año de prolongado distanciamiento, que propició la educación en línea, con los éxitos logrados tal como se evidencia.

Los estudiantes con cierta madurez, logran trabajar a su propio ritmo. La virtualidad favorece su rol de protagonistas en el proceso de aprender. Las herramientas de comunicación on line entre el docente y el estudiante, pueden propiciar aprendizajes colaborativos, enriqueciendo la experiencia con valores añadidos, como el sentido de pertenencia a una comunidad y el cultivo de valores como el respeto, la solidaridad del equipo y el acercamiento a otras culturas.

La modalidad virtual ofrece la posibilidad de comunicarse y manejar distintos formatos y medios, incluso construyendo sus propios recursos, tomar decisiones sobre su 
estrategia y metodología. Generando el desarrollo de habilidades para "aprender a aprender" (Sanabria, 2020).

El aprendizaje virtual implica la idea de mentes activas y complejas, de actores creativos, que aprovechen su conocimiento previo y trabajen de manera comprensiva, llegando a un conocimiento metal a partir del aprendizaje cooperativo. Gracias a las nuevas tecnologías se modifican las relaciones interpersonales, aumenta la comunicación multidimensional y multicultural en el ambiente de aprendizaje. Esto promueve metodologías donde el estudiante es capaz de resolver problemas por sí mismo, sin depender tanto del profesor, ayudándose entre sí y compartiendo información productiva (Yépez Pérez, 2020).

El uso de los Learning Management Systems (LMS), ha sido incorporado crecientemente en la docencia para complementar la clase presencial o introducir modalidades de elearning. Sin embargo, se observa un pobre uso de los LMS para innovar en las propuestas pedagógicas. Un pertinente y estudiando uso de las metodologías de aprendizaje en red, podría contribuir en el uso de los LMS para el diseño de entornos virtuales de aprendizaje (EVA) innovadores, que den vida a modelos pedagógicos que favorezcan el aprender haciendo, dando cabida a diferentes estilos de aprendizaje y generar en los estudiantes competencias asociadas al aprendizaje autónomo, en red y colaborativo. Se requiere un cambio metodológico que permita transitar de un método centrado en el contenido y el profesor, a uno centrado en las e-actividades y el alumno (Silva, 20I7).

Frente a las deficiencias notables de la calidad en la educación, es imperativo emprender acciones que contribuyan a reducir la brecha con los países desarrollados, motivando a que el Estado invierta en materia de TIC, como soporte de los procesos formativos, en las diversas regiones del país (Yong, Nagles, Mejía, \& Chaparro, 2017).

Los docentes universitarios necesitan capacitación tecnológica continua, para alcanzar la competencia digital e integrar de forma coherente las TIC en su función docente (UNEMI, 2018). El constructivismo social expone que el ambiente de aprendizaje óptimo, es aquel donde existe una interacción dinámica entre los expertos, los alumnos y las actividades, lo cual provee oportunidades para que los estudiantes puedan crean su propia verdad, gracias a la interacción con otros y apoyados en las herramientas tecnológicas de la comunicación y la información.

La contraparte de este enfoque, respondería a la evaluación que hacen los docentes respecto a los resultados de aprendizaje de sus estudiantes. La eficiencia o ineficiencia del proceso educativo, tan solo será demostrado con el tiempo, con la evidencia de adquisición de competencias profesionales y humanas por parte del futuro egresado universitario.

\section{REFERENCIAS}

Álvarez de Zayas, C. (1999). La escuela en la vida. Didáctica. La Habana: Pueblo y Educación.

Barros-Bastidas, C., \& Gebera, O. T. (2020). Training in research and its incidence in the 
scientific production of teachers in education of a public university of Ecuador. Publicaciones de La Facultad de Educacion y Humanidades Del Campus de Melilla, 50(2), I67-I85. https://doi.org/I0.30827/publicaciones.v50i2.I3952

Carbajal L., D. (2020). Transición a cursos virtuales y representaciones de la docencia universitaria: la mirada de la comunidad estudiantil del CULagos, marzo-junio 2020. Centro Universitario de los Lagos. Universidad de Guadalajara, 19.

CEPAL-UNESCO. (2020). La educación en tiempos de la pandemia de Covid-19. Santiago de Chile: Naciones Unidas.

De Luca, M. (2020). Las aulas virtuales en la formación docente como estrategia de continuidad pedagógica en tiempos de pandemia. Usos y paradojas. Formación virtual, 33.

Flores, A., Díaz B., F., \& Rigo, M. (2016). Construcción de buenas prácticas educativas mediadas por tecnología. Puebla-México: Facultad de Ciencias de la Electrónica. Benemérita Universidad Autónoma de Puebla.

Gamboa, M., Barros, L., \& Barros, C. (2019). Childhood Aggressiveness, Learning and Self-Regulation in Primary Students. Luz. Revista Electrónica Trimestral de La Universidad de Holguín, 53(9), 1689-1699. https://luz.uho.edu.cu/index.php/luz/article/view/743/637

Hernández, R., \& Infante, M. E. (20I6). El método de enseñanza-aprendizaje de trabajo independiente en la clase encuentro: recomendaciones didácticas. Foro pedagógico, 2I5-23I.

Herrera R., J. I. (2008). El profesor tutor en el proceso de universalización... La Habana: Edit. Universitaria del MES.

IESALC. (2020). Covid-I9 y educación superior: De los efectos inmediaatos a día después. Instituto Internacional de la UNESCO para la Educación Superior de AL y el Caribe, 44.

López, R., Chou, R., \& Granado, J. (2017). La inclusión educativa en la educación a distancia mediante plataformas gestoras. 3er. Congreso Internacional de Ciencias Pedagógicas. Instituto Superior Tecnológico Bolivariano de Tecnología., I07-I I5.

PUCE. Pontificia Universidad Católica del Ecuador. (I2 de 8 de 2020). Medidas académicas. PUCE. Obtenido de https://puceapex.puce.edu.ec/conexionpuce/wpcontent/uploads/2020/08/Medidas-Academicas-de-Flexibilidad-para-el-periodo2020-02-I2-de-agosto_Lineamientos-I.pdf

Rafael S., E. (20I3). Impacto del uso de las TIC en el proceso de autoevaluación con fines de acreditación de carreras universitarias. En J. Dominguez, \& C. Rama, La 
Educación a Distancia en el Perú. (pág. 352). Chimbote: Universidad Católica Los Ángeles .

Sanabria, I. (2020). Educación virtual: Oportunidad para "aprender a aprender". Análisis Carolina. Serie: formación virtual., 42.

Silva, J. (2017). Un modelo pedagógico virtual centrado en la E-actividades. RED. Revista de Educación a Distancia. No. 53. Art. 10, 20.

UNEMI. (2018). Modelo pedagógico de las carreras en línea. Milagro: Universidad Estatal de Milagro.

UNIR. (s.f.).

Yépez Pérez, L. (2020). El trabajo co-operativo y colaborativo en la construcción del conocimiento. Educación virtual. Reflexiones y experiencias., 92.

Yong, E., Nagles, N., Mejía, C., \& Chaparro, C. (2017). E.volución de la educación superior a distancia: desafíos y oportunidades para su gestión. Revista Virtual Universidad Católica del Norte- Colombia, 8I-105. 